Efeito da etillefirina sobre a função cardiorrespiratória de cães anestesiados pelo tiopental
e halotano e pré-tratados ou
não pela levomepromazina

- Etilephrine effects on the cardiorespiratory function of dogs anesthetized by thiopental and halothane, pretreated or not with methotrimeprazine

\author{
* Paulo Cesar de Carvalho Ferreira ${ }^{1}$ - CRMV-SP - n 5441 \\ Flávio Massone ${ }^{2}$ - CRMV-SP - n 0263 \\ Valéria Nobre Leal de Souza Oliva ${ }^{3}$ - CRMV-SP - $\mathrm{n}^{0} 7718$ \\ Francisco José Teixeira Neto ${ }^{2}$ - CRMV-SP - ${ }^{0} 7305$ \\ Stelio Pacca Loureiro Luna ${ }^{2}$ - CRMV-SP - $\mathbf{n}^{0} 4 \mathbf{4 2 0}$ \\ Constanza Sevá Nogueira ${ }^{4}$ - CRMV-SP - $\mathrm{n}^{0} 9899$ \\ Guilherme Jordão de Magalhães Rosa ${ }^{5}$ CRMV-SP - $\mathrm{n}^{0}$ 1000/L \\ Valéria Dus ${ }^{6}$ - CRMV-SP - n ${ }^{0} 5213$ \\ 1 Médico Veterinário do Jockey Club de São Paulo e Pós-graduando de Cirurgia Veterinária - FMVZ/UNESP/Botucatu/SP. \\ 2 Docentes do Departamento de Cirurgia e Anestesiologia Veterinária - FMVZ/UNESP/Botucatu/SP. \\ 3 Docente do Departamento de Clínica, Cirurgia e Reprodução Animal - FOA/UNESP/Araçatuba/SP. \\ Departamento de Cirurgia e Anestesiologia Veterinária UNESP/Botucatu/SP. \\ 5 Docente do Instituto de Biociências - UNESP/Botucatu/SP. \\ 6 Médica Veterinária Autônoma/SP
}

*Jockey Club de São Paulo

Divisão de Assistência Veterinária

Rua Bento Frias n 248 - Butantā

CEP 05423-050 - São Paulo - SP.

Fone: $(0 * \star 11) 3816-4011 /$ r. 298

End. Eletrôn.: dav.jockey@hipernet.com.br

\title{
RESUMO
}

A etilefrina, agente simpatomimético agonista alfa, beta- 1 e beta-2, de longa duração, foi estudada em 40 cães, machos e fêmeas, distribuídos eqüitativamente em 4 grupos, pré-tratados ou não pela levomepromazina, sob a anestesia pelo tiopental e halotano, nos quais se procurou avaliar a função cardiorrespiratória. Nos animais não pré-tratados pela levomepromazina, quando tratados pela etilefrina (Grupo II), em relação ao seu controle (Grupo I), observou-se que a frequiência cardíaca (FC), a pressão arterial sistólica (PAS), a pressão arterial média (PAM), a pressão arterial diastólica (PAD) e a pressão venosa central (PVC) aumentavam significativamente $(\mathrm{p}<0,05)$, enquanto que no grupo pré-tratado pela levomepromazina e que recebiam etilefrina (Grupo IV), em relação ao seu controle (Grupo III), observou-se que a frequiência cardíaca aumentava, porém a PAS, a PAM, a PAD e a PVC diminuíam consideravelmente em relação aos valores basais. Quanto ao seu efeito arritmogênico, observou-se que, no Grupo II, alguns animais apresentavam extra-sístoles ventriculares no traçado eletrocardiográfico, após o tratamento com a etilefrina, o que não ocorreu no grupo IV. Além de suas propriedades hemodinâmicas, detectou-se alguma ação analéptica, mediante aumento do volume-minuto, porém não foi significativo $(\mathrm{p}<0,05)$.

Palavras-chave: etilefrina, levomepromazina, tiopental, halotano. 
FERREIRA, P.C.C.; MASSONE, F; OLIVA, V.N. L. S.; TEIXEIRA NETO,F. J.; LUNA, S. P. L.; NOGUEIRA, C. S.; ROSA, G. J. M.; DUS, V. Efeito da etilefrina sobre a função cardiorrespiratória de cães anestesiados pelo tiopental e halotano e pré-tratados ou não pela levomepromazina / Etilephrine effects on the cardiorespiratory function of dogs anesthetized by thiopental and halothane, pretreated or not with methotrimeprazine / Rev. educ. contin. CRMV-SP / Continuous Education Journal CRMV-SP, São Paulo, volume 4, fascículo 3, p. 76 - 83, 2001.

\section{Introdução e Literatura}

Anestesiologia Veterinária recebeu grande incentivo com a descoberta de SUCKLING; RAVENTOS (1956) apud JONES, 1993 de um agente anestésico volátil, não inflamável e potente, o halotano.

Estudos mais recentes demonstram que o halotano deprime a contratilidade do miocárdio, a freqüência e débito cardíaco, quando se eleva a concentração alveolar mínima (BOYOD et al., 1991), consequentemente diminuição da pressão arterial, sugerindo depressão do barorreflexo (BARASH et al., 1991).

O tratamento com fármacos vasoativos para corrigir a depressão do miocárdio induzida pelo halotano torna-se indispensável para que a pressão arterial volte a valores basais (TARNOW et al., 1973).

A etilefrina é uma amina simpatomimética introduzida na Europa Central e Escandinávia por SPITZBARTH (1950). Possui ação mista beta-1 e alfa, e em menor intensidade beta-2, exibindo efeito inotrópico positivo e em menor grau efeito cronotrópico VOLKER (1994). Possui ainda, um outro mecanismo, o da ação direta constatado por WESTERMANN; ROEHRS (1980).

A etilefrina deprime intensamente o reflexo da vasodilatação periférica, que ocorre comumente com os fármacos de ação indireta após a liberação de noradrenalina, aumentando desta forma consideravelmente o seu efeito WELLENS; SZIGETVARI (1968).

CARREIRA (1977), analisando o retorno venoso, a força de contração cardíaca, a freqüência cardíaca e a pressão arterial, em estudos comparativos da etilefrina com outros fármacos vasoativos, como a dopamina, em cães anestesiados pelo pentobarbital sódico, concluiu que a dopamina apresentava maior efeito sobre a contratilidade e resistência vascular, ao passo que a etilefrina possuía ação superior no retorno venoso.

A etilefrina utilizada durante a depressão do miocárdio $(0,045 \mathrm{mg} / \mathrm{Kg}, \mathrm{IV}$, por 10 segundos $)$ foi qualificada como medicação de suporte para manter a pressão de perfusão aos órgãos vitais, por TARNOW et al. (1973), que observou, ainda, extra-sístoles ventriculares em dois cães de um grupo de treze animais. O seu efeito máximo iniciava-se aos três minutos após aplicação e perdurava por mais de trinta minutos.

O halotano e o metoxifluorano causam depressão da automaticidade do nódulo sinusal dose-dependente, além de sensibilizar o miocárdio à ação das catecolaminas, causando severas arritmias ventriculares dose-dependentes, que podem ser controladas com a diminuição da concentração do agente anestésico; porém, outras alterações, não só aquelas induzidas pelo halotano, como modificações no tônus vagal e simpático, bem como distúrbios eletrolíticos e ácido-base, poderão contribuir no aparecimento das arritmias. $\mathrm{O}$ agente anestésico, bem como a intubação traqueal e as manipulações cirúrgicas, também podem provocar arritmias (TILLEY, 1992).

\section{Material e Método}

Foram utilizados quarenta cães sem raça definida, machos e fêmeas, com peso de 9,0 a $17,5 \mathrm{Kg}$, considerados sadios, evitando-se fêmeas em cio, cães irascíveis ou animais provenientes de outros experimentos. Instituiuse jejum prévio de 12 horas e hídrico de 4 horas sendo todos eles provenientes do Biotério Central da UNESP Campus de Botucatu -SP.

Os 40 cães utilizados foram distribuídos aleatória e eqüitativamente em 4 grupos, conforme protocolo anestésico, discriminado a seguir:

GRUPO I (G I): os animais receberam o placebo (Cloreto de Sódio a 0,9\%, 0,2 ml/Kg, IV) e, decorridos 15 minutos, aplicou-se, para indução da anestesia, tiopental sódico (25 mg/Kg, IV) e, para mantê-la utilizou-se halotano.

GRUPO II (G II): utilizou-se o mesmo procedimento que G I, porém, decorridos 45 minutos do início do experimento, aplicou-se a etilefrina $(0,05 \mathrm{mg} / \mathrm{kg}$, IV, durante 1 minuto);

GRUPO III (G III): os animais receberam levomepromazina (1mg/Kg, IV) e, após 15 minutos, aplicouse tiopental sódico para indução da anestesia (12,5 mg/ $\mathrm{Kg}$, IV) e, para mantê-la, usou-se o halotano. Decorridos 45 minutos do início do experimento, aplicou-se o placebo (Cloreto de Sódio a 0,9\%, 0,05 ml/Kg, IV durante 1 minuto;

GRUPO IV (G IV): os animais receberam o mesmo tratamento do grupo III, substituindo-se o placebo pela etilefrina, na mesma dose e via de G II.

As mensurações paramétricas foram feitas nos instantes (em minutos) 0, 15, 30, 45 (M1), 47 (M2), 50 (M3), 55 (M4), 60(M5) e 70 (M6) e anotadas em uma planilha, na seguinte ordem: frequiência cardíaca (FC), em batimentos por minuto; freqüência respiratória (f), em movimentos por minuto; volume-corrente $\left(\mathrm{V}_{\mathrm{T}}\right)$, em litros; $\mathrm{ETCO}_{2}$, em $\mathrm{mmHg} ; \mathrm{SPO}_{2}$, em percentagem; 


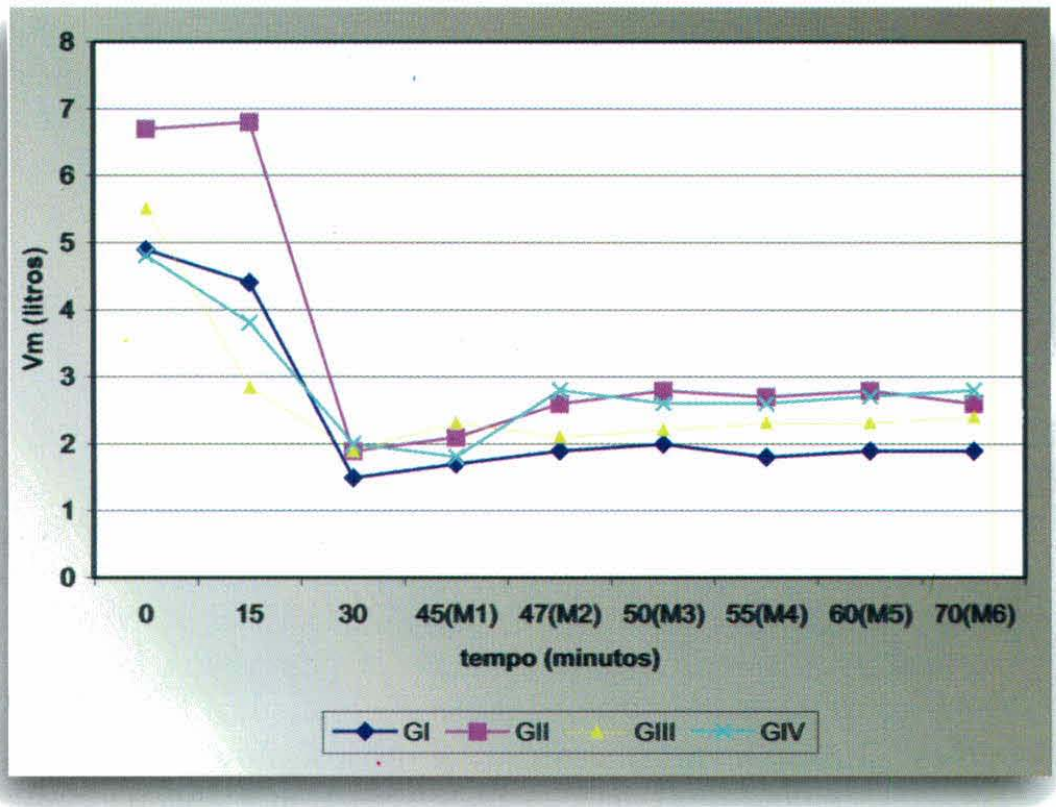

Figura 1. Variação dos valores médios do Vm, em litros, em animais anestesiados pelo tiopental e halotano e tratados ou não pela etilefrina e pré-tratados ou não pela levomepromazina, em diferentes momentos.

pressões arteriais sistólica (PAS), média (PAM) e diastólica (PAD), em mmHG (antes da anestesia, empregou-se o método não invasivo - oscilométrico - e, após, empregou-se o método invasivo); pressão venosa central (PVC), em cm de $\mathrm{H}_{2} \mathrm{O}$; pela hemogasometria foram avaliados a $\mathrm{PaO}_{2}$ e a $\mathrm{PaCO}_{2}$, em $\mathrm{mmHg}$; o pH, em logaritmo; o BI, em mEq/l e o EB, em mEq/l; ainda, anotaram-se a temperatura retal, em graus Celsius, e a eletrocardiografia (ECG), em D II.

A análise estatística foi efetuada utilizando-se a Análise Multivariada de Perfis (MORRISON, 1991; ROSA, 1994), com quatro grupos experimentais e seis ou três momentos de mensuração, dependendo da variável considerada.

\section{Resultados e Discussão}

A etilefrina, atua indiretamente sobre a função cardiovascular a partir do efluxo de noradrenalina (FROST et al., 1978) e diretamente sobre os receptores beta e alfa WESTERMANN; ROEHRS (1980) com atuação preferencial sobre os receptores do tipo beta-1 adrenérgicos e pouco efeito sobre os receptores alfa (EKESTROM et al., 1980).

Quanto a ventilação a etilefrina exibiu um comportamento atípico no volume minuto (Figura 1), pois foram observadas algumas diferenças entre os momentos e grupos, mesmo que não detectadas estatisticamente.

Esses resultados são conflitantes com os obtidos por outros autores que, utilizando a etilefrina, não observaram alteração na freqüência respiratória em cães anestesiados pelo pentobarbital sódico (AQUINO, 1968) e pelo halotano (TARNOW et al., 1973). HAPKE (1964) foi o único autor a mencionar um efeito não específico da etilefrina, ao observar uma atividade analéptica respiratória, quando relatou frequiência respiratória inalterada, mas volumes corrente e minuto aumentados. No presente experimento, $60 \%$ dos cães de G II e $80 \%$ dos cães em G IV apresentaram essa atividade analéptica sobre o sistema respiratório.

O aumento da FC (Figura 2), após o uso da etilefrina, foi significativo em G II e G IV ( $p<0,05)$, deduzindo-se, assim, que, mesmo com o bloqueio alfa da levomepromazina, a ação cronotrópica, quando da estimulação beta da etilefrina, fez-se presente.

É sabido que o efeito da etilefrina sobre a freqüência cardíaca é muito variável, podendo aumentála, diminuí-la (CARREIRA, 1977; HEIDRICH, 1977; HAHN, 1980; CYONG, 1982) ou mesmo mantê-la inalterada (AQUINO, 1968). A ação cronotrópica provavelmente deve ter ocorrido pelo estímulo direto nos receptores beta-1 no coração, baseando-se pelo homem (ORTENZI; TARDELLI, 1996); porém, existe alguma evidência do efeito cornotrópico ser alfa-mediado e foi defendida por WALKENHORST et al. (1981), que, estudando em cães a diferenciação dos receptores alfa e beta cardíacos e periféricos em resposta à etilefrina e outros fármacos vasoativos, con-

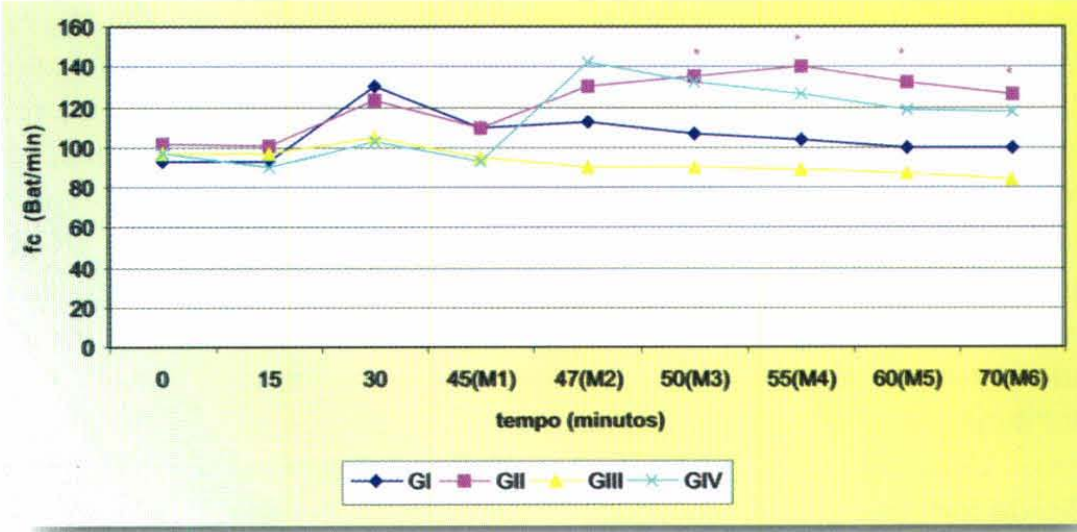

Figura. 2. Variação dos valores médios do FC, em batimentos por minuto, em animais anestesiados pelo tiopental e halotano e tratados ou não pela etilefrina e pré-tratados ou não pela levomepromazina, em diferentes momentos. 
cluiu que a ação cronotrópica da etilefrina originava-se da estimulação alfa do miocárdio e do nódulo sino-atrial, uma vez que essa ação era abolida pelo tratamento com a fenoxibenzamina, um bloqueador alfa. Essa abordagem vem contrariar o nosso conceito, baseado no humano, sobre a ação cronotrópica cardíaca, porém o mapeamento dos receptores cardíacos e periféricos difere muito nas diferentes espécies, sugerindo a existência de uma pequena população de receptores alfa-adrenérgicos no nódulo sinusal canino (WALKENHORST et al. 1981).

Observou-se também que a freqüência cardíaca não diminuiu após a aplicação da etilefrina, mecanismo este comum no uso dos sim-

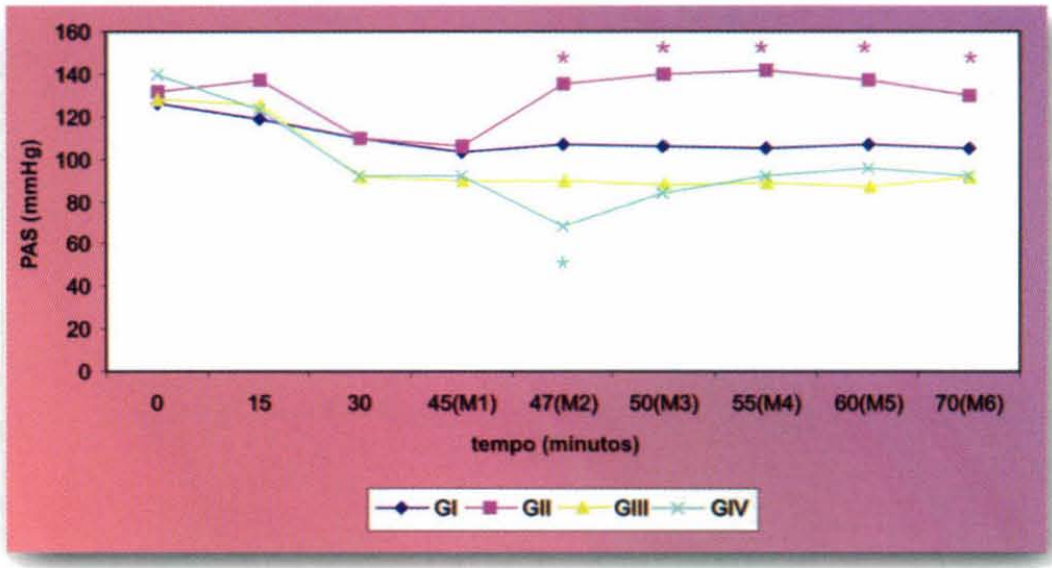

Figura 3. Variação dos valores médios da PAS em mmHG em animais anestesiados pelo tiopental e halotano e tratados ou não pela etilefrina e pré-tratados ou não pela levomepromazina, em diferentes momentos. patomiméticos de ação mista, onde a liberação de noradrenalina (ação indireta) poderá aumentar subitamente a pressão e desencadear bradicardia e vasodilatação periférica via baroreflexo, possivelmente a etilefrina deprima este reflexo, também observada por WELLENS; SZIGETVARI (1968). Ainda dando ênfase a atividade dos barorreceptores sabe-se que o halotano prejudica a sua atividade frente as diminuições da pressão arterial e freqüência cardíaca (BARASH et al., 1991).

Nos grupos II e IV não houve uma diminuição da freqüência cardíaca, o que é comum quando do uso dos simpatomiméticos de ação mista, pelo seu mecanismo de ação indireta - observado também por WELLENS; SZIGETVARI (1968) - possivelmente deprimindo o barorreflexo, desencadeado pela liberação de noradrenalina, que pode aumentar subitamente a pressão e provocar a bradicardia e vasodilatação periférica. Ainda, dando ênfase à atividade dos barorreceptores, sabe-se que o halotano prejudica a sua atividade mediante as diminuições da pressão arterial e da freqüência cardíaca (BARASH et al., 1991).

A PAM (Figura 4) mostrou comportamento simi- que, após administrar-lhe intravenosamente, sua concentração plasmática apresenta uma atividade bioexponencial e a sua meia vida exibe uma fase alfa com duração de 6,2 minutos e uma fase beta de até 2,2 horas, segundo VOLKER (1994). Dessa forma, fica claro que a PAS aumentou consideravelmente no grupo não pré-tratado com a levomepromazina (G II), pois apresentou uma resposta alfa inicial que provavelmente esteja relacionada com a venoconstrição e aumento do retorno venoso, fato observado por CARREIRA (1977), e/ou por um efeito sinérgico do mecanismo de ação indireto, que atuaria inicialmente liberando noradrenalina (FROST et al., 1978).

Decorridos os primeiros 6-7 minutos da fase alfa, sucedeu provavelmente a fase beta, responsável pelo principal efeito hemodinâmico da etilefrina, a ação inotrópica (CARREIRA, 1977).

Em G II, não houve bloqueio alfa, pois a pressão arterial aumentou consideravelmente após o aplicação da etilefrina, o que leva a crer que houve uma diminuição da capacitância dos vasos (venoconstrição), mecanismo esse proposto por TARNOW et al. (1973). lar à PAS (Figura 3) nos grupos estudados, apresentando um aumento intenso em G II, já após 2 minutos da aplicação da etilefrina, porém somente foi significativo, em ambas, aos 55 minutos (M4).

A PAD (Figura 5), embora demonstre, após visualização gráfica, um comportamento similar à PAS e PAM, não apresentou efeito de momento e, sim, apenas valores superiores para o G II.

A explicação para o comportamento da PAS e PAM relaciona-se muito à atividade farmacodinâmica da etilefrina, pois se sabe

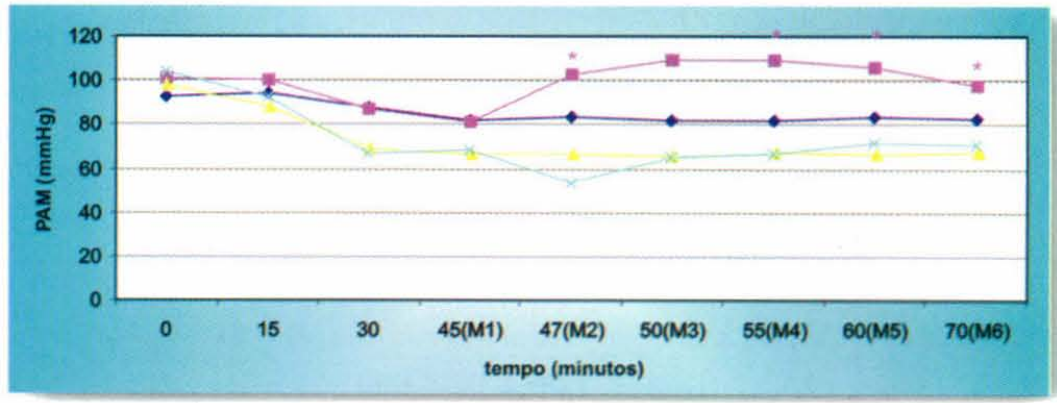

Figura 4. Variação dos valores médios da PAM em mmHG em animais anestesiados pelo tiopental e halotano e tratados ou não pela etilefrina e pré-tratados ou não pela levomepromazina, em diferentes momentos. 


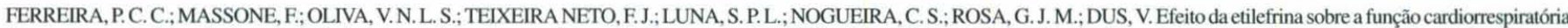

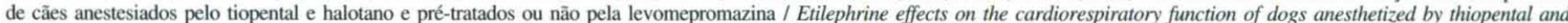

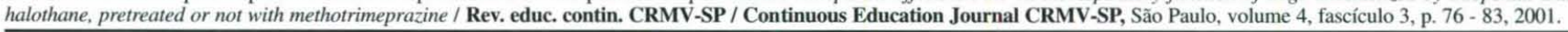

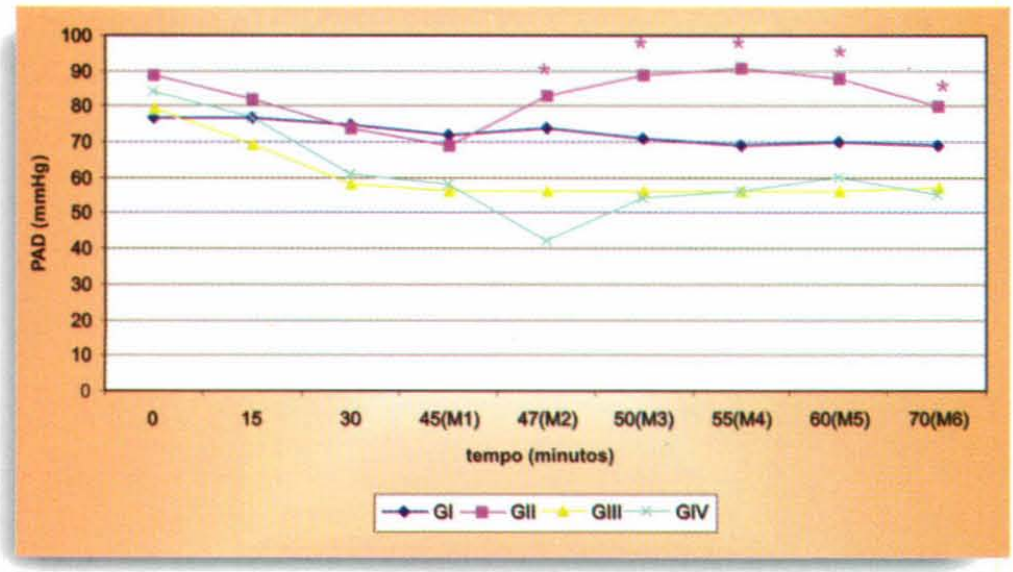

Figura 5. Variação dos valores médios da PAD em mmHG em animais anestesiados pelo tiopental e halotano e tratados ou não pela etilefrina e pré-tratados ou não pela levomepromazina, em diferentes momentos.

A PAD aumentou consideravelmente após a aplicação da etilefrina em GII nos primeiros cinco minutos de atuação, sem sair dos parâmetros fisiológicos e, posteriormente, declinou de forma lenta até os 70 minutos. TARNOW et al. (1973) avaliou a PAD e considerou o seu discreto aumento como decorrente da ação alfa-adrenérgica sobre as veias, produzindo uma venoconstrição, um aumento no retorno venoso e a elevação do enchimento ventricular, pois essa ação era revertida ao usar um alfabloqueador, a fenoxibenzamina.

Já no grupo pré-tratado com a levomepromazina (G IV), a PAD diminuiu nos primeiros 47 minutos (M2), retornando ao valor anterior ao tratamento, após $50 \mathrm{mi}$ nutos de sua aplicação e, assim, manteve discreta tendência a aumentar até os 70 minutos (M6). Essa ação bloqueadora da levomepromazina sobre G IV foi análoga à obtida pela fenoxibenzamina.

A PVC (Figura 6) em G II (tratado com a etilefri- (sistema circulatório porta) e do fluxo sangüíneo arterial para o fígado - e a responsável por até $29 \%$ do débito cardíaco no homem (GUYTON; HALL 1996).

As áreas de estoque do fígado, sobretudo o parênquima, e, em menor grau, os sinusóides, sofrem constrição pela estimulação simpática, ejetando sangue para a circulação geral de um a quatro minutos no homem, o que significa até $350 \mathrm{ml}$ de sangue.

A estimulação simpática tem efeito direto praticamente em todos os vasos sangüíneos do sistema gastrointestinal, ocasionando vasoconstrição intensa até que um mecanismo controlador de fluxo "escape auto-regulatório" produza vasodilatação, fornecendo também volume auxiliar à volemia. $\mathrm{O}$ baço apresenta uma cápsula que sofre influência da estimulação simpática capaz de produzir-lhe uma contração bem como de seus vasos, responsável pelo aumento de 3 a 4\% do hematócrito sangüíneo, constituindo, assim, uma ajuda ao organismo durante períodos de estresse circulatório, garantindo o retorno venoso, conforme citou GUYTON; HALL (1996).

As alterações eletrocardiográficas (Figuras 7 e 8 ) fizeram-se presentes principalmente em G II, no qual se verificou um ritmo ventricular ectópico, compatível com a contração ventricular prematura (CVP) ou também denominada de extra-sístole ventricular, que era isolada em M2 e M3, porém, em M4, ou seja, 10 minutos após a aplicação da etilefrina, essa anomalia surgiu com uma intensidade maior. Enumeram-se como causas predisponentes dessa arritmia a técnica anestésica empregada, o plano superficial da anestesia, o distúrbio do equilíbrio na) apresentou aumento considerável e foi significativo ( $\mathrm{p}<0,05)$ aos 47 minutos (M2) e 50 minutos (M3), diminuindo posteriormente até aos 70 minutos (M6), aproximando-se dos valores antes do tratamento. Esse aumento na PVC traduz-se em melhoria nos parâmetros hemodinâmicos em face da redistribuição sangüínea da região esplâncnica para a circulação venosa, fato este muito bem evidenciado por VOLKER (1994). Ao se mensurar a PVC, avaliou-se indiretamente o retorno venoso, a pressão de enchimento do ventrículo direito ou a função do ventrículo direito.

A importância da pré-carga procedente da venoconstrição não é o único mecanismo desencadeado pela etilefrina, pois existe também a diminuição da capacitância dos vasos da circulação esplâncnica - composta por vasos do intestino, baço, sistema porta venoso, fígado

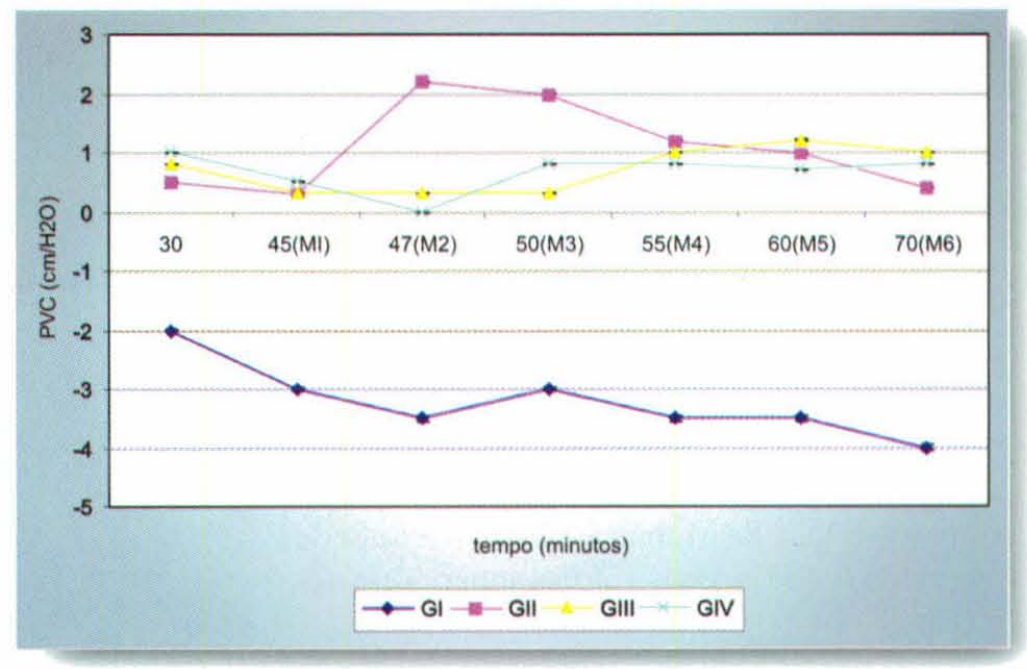

Figura 6. Variação dos valores médios da PVC em cm $\mathrm{H}_{2} \mathrm{O}$ em animais anestesiados pelo tiopental e halotano e tratados ou não pela etilefrina e pré-tratados ou não pela levomepromazina, em diferentes momentos. 
ácido-básico e eletrolítico, a hipoxemia e a sensibilização do miocárdio pelas catecolaminas (ETTINGER, 1983). O mecanismo de formação dessa anomalia pode estar associado a um desequilíbrio do tônus autonômico (MUIR, 1977).

Uma vez que o Grupo II não foi pré-tratado com a levomepromazina (bloqueador alfa), a estimulação alfa da etilefrina encontrou provavelmente papel preponderante no desencadeamento dessa arritmia, uma vez que o nódulo sino-atrial tem uma importância no controle do cronotropismo (WALKENHORST et al. 1981). Outros estudos confirmaram essa hipótese de que sensibilização do miocárdio à ação das catecolaminas ocorrera primariamente na porção pós-sináptica dos receptores alfa do miocárdio e, com menor intensidade, na dos receptores beta-1 (MAZE; SMITH, 1983). Analisando essa alteração arrítmica com a farmacodinâmica da etilefrina, uma vez que a fase de estimulação alfa apresenta efeito nos primeiros 6 minutos, neste estudo, prolongou-se aproximadamente $10 \mathrm{mi}$ nutos. Dessa forma, pôde-se correlacionar a estimulação alfa dos receptores do miocárdio com a presença da extra-sístole ventricular, após a aplicação da etilefrina.

\section{ELETROCARDIOGRAFIA (ECG)}

Observou-se que o traçado eletrocardiográfico nos grupos I, III e IV foi compatível com a normalidade nos momentos avaliados (M1, M2, M3, M4, M5, M6), porém, em G II, detectou-se alteração no ritmo sinusal.

M1. o ritmo exibido nos quatro grupos foi o sinusal, alterada apenas a freqüência cardíaca, que estava aumentada em G II ao comparar-se com o seu controle (G I);

M2. detectou-se, já neste momento, dois minutos da aplicação da etilefrina, a presença de taquicardia paroxística multifocal, acompanhada de uma arritmia sinusal compatível com extra-sístole ventricular ou contração ventricular prematura (VPC), em G II. Entretanto, no grupo pré-tratado com a levomepromazina (G IV), a freqüência cardíaca aumentou, porém o ritmo permaneceu sinusal (Figura 7);

M3. a freqüência cardíaca apresentou-se normal nos quatro grupos, porém, em G II, persistiram algumas extra-sístoles ventriculares isoladas, enquanto que, em G IV, o ritmo permaneceu sinusal e sem alterações;

M4. decorridos 10 minutos da aplicação da etilefrina, observou-se uma seqüência de repetições de extra-

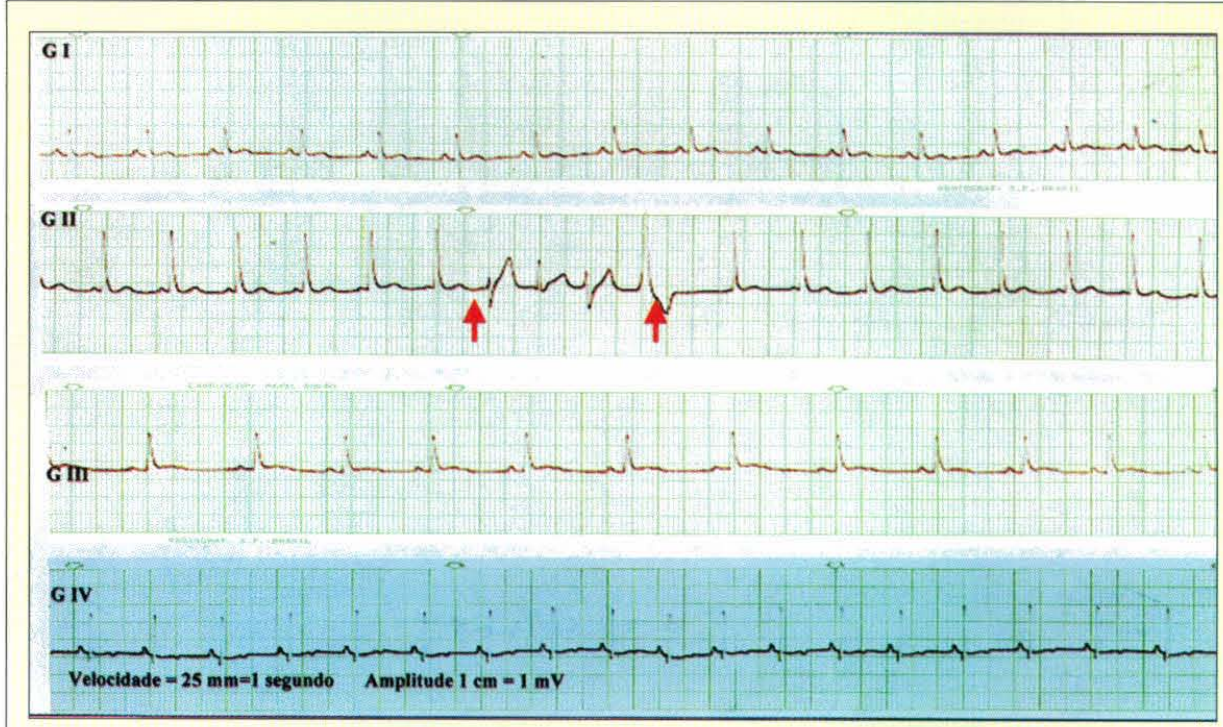

Figura. 7 - Apresentação simultânea dos grupos GI, G II, G III e G IV , 2 minutos após a aplicação de placebo ou etilefrina (M2).Observar a taquicardia paroxística multifocal em G II (setas vermelhas).

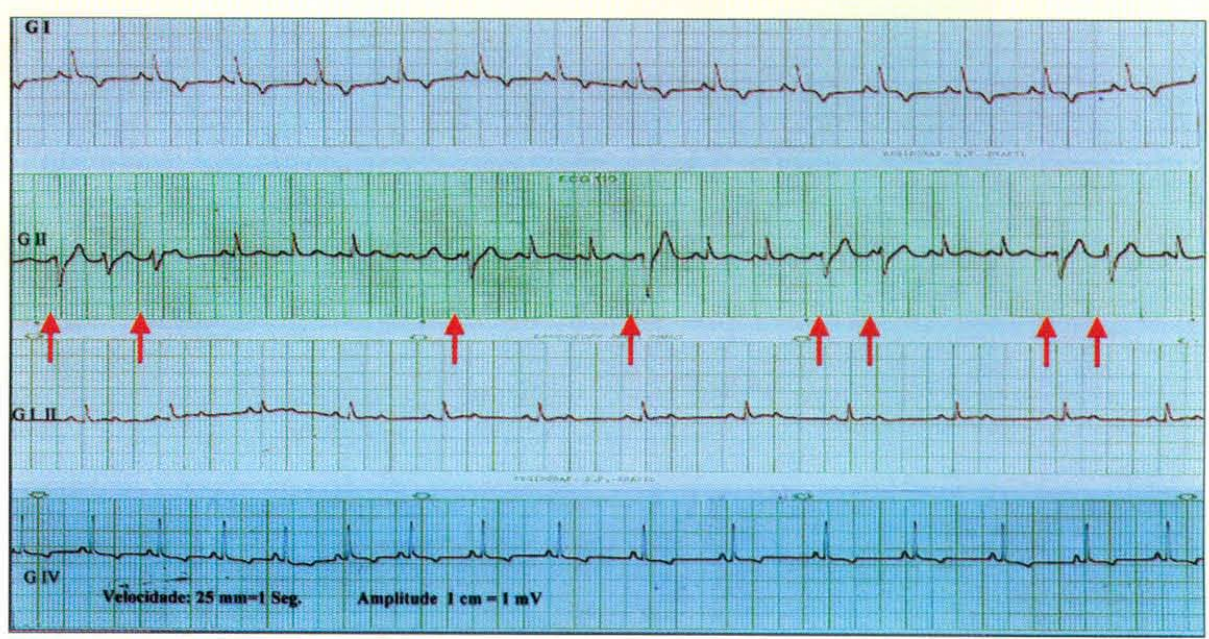

Figura 8. Apresentação simultânea dos grupos GI, G II, G III e G IV , 5 minutos após a aplicação de placebo ou etilefrina (M4).Observar o aumento as extrassítoles (setas vermelhas). 
sístoles ventriculares em G II, enquanto que, nos demais grupos, o ritmo foi o sinusal (Figura 8);

M5. o ritmo permaneceu inalterado neste momento, porém com taquicardia em $\mathrm{G}$ II;

M6. o ritmo apresentou-se sinusal nos quatro grupos estudados; no entanto, em G I (grupo controle), apresentou-se com taquicardia.

\section{Conclusões:}

Diante do desenvolvimento experimental, é permitido concluir que:

1. a levomepromazina causou discreta bradicardia em alguns cães, em razão do bloqueio dos receptores alfa do nódulo sino-atrial;

2. A etilefrina leva a uma discreta hipertensão arterial (sistólica, média e diastólica), acompanhada de taquicardia intensa nos grupos não pré-tratados com levomepromazina;
3. A ação analéptica da etilefrina aumentou o volumeminuto, fenômeno este que, apesar de não ser significativo, requer estudos mais detalhados;

4. A etilefrina mostrou sua ação sobre o retorno venoso em G II (sem bloqueio alfa), caracterizando o seu mecanismo venoconstritor, que foi possível visualizálo graças ao monitoramento da pressão venosa central;

5. O bloqueio alfa da levomepromazina foi importante na proteção do miocárdio quanto à arritmia, porém deve-se avaliar a dose e tempo empregados, pois esses tranqüilizantes anulam a venoconstrição da etilefrina, mecanismo importante no tratamento da hipotensão oferecido pelos fármacos de ação mista;

6. Animais pré-tratados pela levomepromazina e anestesiados pelo tiopental e halotano, quando submetidos ao tratamento da etilefrina, não sofrem alterações de ritmo e amplitude cardíaca, quando comparados ao seu controle (G II).

\section{SUMMARY}

A long duration sympathomimetic agent, alfa-1, beta- 1 and beta- 2 agonist, etilephrine was studied in 40 dogs, males and females, divided into four groups. The cardiorespiratory function was evaluated under thiopental and halothane anesthesia, with or without etilephrine administration and with or without methotrimeprazine pretreatment. It was found that heart rate (HR), systolic blood pressure (SBP), average blood pressure (ABP), diastolic blood pressure (DBP) and central venous pressure $(\mathrm{CVP})$ were significantly increased $(\mathrm{p}<0.05)$ in the group that was not pretreated with methotrimeprazine but received etilephrine (Group II) when compared to their control (Group I). On the other hand, the group of animals pretreated with methotrimeprazine that also received etilephrine (Group IV) displayed increased heart rate but SBP, ABP, DBP and CVP were considerably reduced when compared to their control (Group III). Regarding its arrhythmogenic effect, it was observed that some animals in Group II had ventricular extrasystole after treatment with etilephrine. This was not observed in Group IV. Besides the hemodynamic properties, some analeptic action was detected through the minute volume increase, although it was not significant $(\mathrm{p}<0.05)$.

Key words: etilephrine, methotrimeprazine, thiopental, halothane.

\section{AGRADECIMENTO}

À Boehringer Ingelheim e CAPES pelo auxílio financeiro na execução deste trabalho. 


\section{REFERÊNCIAS}

1. AQUINO, A. B. Aminas Simpatomiméticas de uso corrente. Ação cardio-circulatória em cães normais- observações, O Hospital, v. 74, n. 5, p. 1543-1552, 1968.

2. BARASH, P. G.; CULLEN, B. F.; STOELTING, R. K. Manual de anestesiologia clínica. São Paulo: Manole, 1991. 621 p.

3. BOYOD, C. J.; McDONELL, W. N.; VALLIANT, A. Comparative hemodynamic effects of halothane and halothane-acepromazine at equipotent doses in dogs. Canadian Journal Veterinary Research, v. 55, n. 2, p. 107-112, 1991.

4. CARREIRA, A. L. V. Análise farmacológico de algunos efectos simpatomiméticos en el perro anestesiado. 1977. Tese (Doutorado) - Centro de Investigacíon y de Estudios Avanzados del instituto Politécnico Nacional, México.

5. CYONG, J. C. Comparison of effects of Adrenaline, Ethylephrine, Isoprenaline and Terbutaline on the Vascular System. Arch. Int. Pharmacodyn, p. 255-262, 1982.

6. EKESTROM, S.; LILJEQVIST, L.; NORDHUS, O. The effect of etilefrine (Effortil) on regional blood flow during arterial reconstructive surgery. Thorac. Cardiovas. Surg., v. 28, n. 6, p. 410-413, 1980.

7. ETTINGER, S. J. In:- Textbook of veterinary internal medicine. 2. ed. Philadelphia: W. B. Saunders, 1983. p. 980 .

8. FROST, B. R.; HALLORAN, T. N.; FREWIN, D. B.; GERKE, D. C.; DOWNEY, J. A. The indirect sympathomimetic activity of etilefrine;a comparison with tyramine and ephedrine using H3 noradrenaline. Journal Pharmacy and Pharmacology, v. 30 , n. 10 , p. $628-631,1978$.

9. GETTY, R. Anatomia dos animais domésticos. 5. ed. Rio de Janeiro: Interamericana, 1981. v. 1, p. 108-135.

10. GUYTON, A. C.; HALL, J. E. The Circulation. In:Textbook of medical physiology. 9th ed. Philadelphia: W. B. Saunders, 1996. p. 161-169.

11. HAHN, N. Tierexperimentelle Untersuchungen zur wirkung von Etilefrinhydrochloride auf Herz und Kreislauf bei partiel ischaemiegeschaedigtem myokard. Z. Kardiol., v. 69, p. 771$778,1980$.

12. HAPKE, H. J. Continous Infusion of Cardiovascular Agents. Die Kleintierpraxis, v. 9, n. 6, p. 169-172, 1964.

13. HEIDRICH, H. Animal experimental investigations on the influence of etilefrine upon myocardial contractility. IRCS Med. Sci. Cardiovasc. Syst. Pharmacol., v. 5, p. 270, 1977.
14. JONES, R. S. From hemlock to ramifidine. Equine Veterinary Education, v. 5, n. 4, p. 197-1999, 1993.

15. KRAHL, V. E., 1962 apud GETTY, 1981, p. 108.135.

16. MAZE, M.; SMITH, C. M. Identification of receptor mechanism mediating epinephrine-induced arrythmias during halothane anesthesia in the dog. Anesthesiology, v. 59, p. 322-326, 1983.

17. MORRISON, D. F. Multivariate statistical methods. 3. ed. New York: McGraw Hill, 1991.

18. MUIR, W. W. Thiobarbiturate-induced dysrhytmias: the role of heart rate and autonomic imbalance. American Journal Veterinary Research, v. 38, p. 1377-1381, 1977.

19. ORTENZI, A. V.; TARDELLI, M. A. Sociedade de anestesiologia do Estado de São Paulo. São Paulo: Atheneu, 1996. 934 p.

20. ROSA, G. J. M. Desenvolvimento de aplicativo para a análise de perfil na experimentação zootécnica. 1994. Dissertação (Mestrado) - FMVZ-UNESP-Botucatu, SP.

21. SPITZBARTH, H. Erfahrungen mit Effortil bei arterieller hypotonic. Med. Klin., v. 50, p. 1593-1596, 1950.

22. SUCKLING; RAVENTOS, 1956 apud JONES, R. S. 1993, p. 197-199.

23. TARNOW, J. et al. Haemodynamic responses to ethylphenylephrine (Effortil) during halothane-induced myocardial depression in the dog. Acta.Anaesth.Scand., v. 17, p. 184-189, 1973.

24. TILLEY, L. P. Essentials of canine and feline electrocardiography: Interpretation and treatment. 3. ed. Philadelphia: Lea e Febiger, 1992. 470 p.

25. VOLKER, K. L. Expert Report Treatment of Symptomatic Orthostatic Hypotension With Etilefrine Hydrochloride (Effortil). Germany: Boehringer Ingelheim Gmgh. Corporate Division Medicine, 1994.

26. WALKENHORST, R.; REINHARDT, G.; ARNOLD, G. Differentiaton of Cardiac and Peripheral Alpha and BetaAdrenergic Responses to Dobutamine, Etilefrine and Xylometazoline in Dogs. Pharmacology, v. 22, p. 294-304, 1981.

27. WESTERMANN, K. W.; ROEHRS, M. M. Hemodynamic effects of etilefrine.Dose- related reactions to IA and IV drug application before and after alfa- and beta-blockade. Herz Kreisl., v. 12 , n. 10 , p. $442-452,1980$.

28. WELLENS, D.; SZIGETVARI, E. Effect of tyramine and some adrenergic amines upon reflexogenic vasodilation. Arch. Int. Pharmacodyn., v. 176, n. 2, p. 480-484, 1968. 